\title{
THE BEHAVIOR OF MHD FLOW AND HEAT TRANSFER IN THE PRESENCE OF HEAT SOURCE AND CHEMICAL REACTION OVER A FLAT PLATE
}

\author{
Matthew OluWafemi LaWAL \\ Department of Mathematics \\ Adeyemi College of Education, Ondo, Nigeria. \\ lawalmo@aceondo.edu.ng \\ Suraju Olusegun Ajadi \\ Department of Mathematics \\ Obafemi Awolowo University, Ile-Ife, Nigeria. \\ sajadi@oauife.edu.ng
}

[Received: March 23, 2016, Accepted: August 10, 2016]

\begin{abstract}
This article is on a study of the behavior of MHD flow and heat transfer in the presence of heat source and chemical reaction over a flat plate. The steady two dimensional partial differential governing equations are transformed using standard dimensionless variables resulting in a coupled nonlinear ordinary differential equation with some embedded parameters. The Homotopy Perturbation Method (HPM) is employed to solve the system of dimensionless equations and the approximate analytical solutions obtained are further analyzed using MAPLE 17 symbolic platform. It is observed that the flow velocity within the boundary layer decreases with increasing magnetic field. Further parametric analysis shows that the temperature increases with increasing Hartmann number while it decreases with increasing Prandtl number. Further observation reveals that temperature profile increases rapidly with increasing heat source and chemical reaction parameters while the concentration profile decreases with increasing heat source and chemical reaction parameters. Graphical demonstrations of these solutions shed more lights on the behavior of the system.
\end{abstract}

Mathematical Subject Classification: 05C38, 15A15

Keywords: chemical reaction, heat source, HPM, MHD boundary layer, flat plate.

\section{Nomenclature}
a $[1 / \mathrm{s}]$
stretching rate,
$B_{o} \quad[-]$
applied uniform transverse magnetic field strength, (magnetic induction)
$C \quad\left[\mathrm{~mol} / \mathrm{m}^{3}\right]$
dimensional species concentration of the fluid,
$C_{p} \quad[\mathrm{~J} /(\mathrm{kg} \mathrm{K})] \quad$ specific heat capacity at constant pressure,
$C_{w} \quad\left[\mathrm{~mol} / \mathrm{m}^{3}\right] \quad$ species concentration of the fluid along the sheet wall,
$C_{\infty} \quad\left[\mathrm{mol} / \mathrm{m}^{3}\right]$ species concentration of the fluid far away from the sheet wall,
$D \quad\left[\mathrm{~m}^{2} / \mathrm{s}\right]$ effective diffusive coefficient or mass diffusion coefficient, 


\begin{tabular}{|c|c|c|}
\hline$F$ & -1 & fuel, \\
\hline$M$ & - & Hartmann number, \\
\hline$P$ & {$\left[\mathrm{~N} / \mathrm{m}^{2}\right]$} & pressure, \\
\hline $\operatorname{Pr}$ & {$[-]$} & Prandtl number, \\
\hline$Q$ & {$[\mathrm{~J}]$} & internal heat generation term, \\
\hline$Q_{o}$ & {$[\mathrm{~J}]$} & dimensional heat absorption or generation coefficient, \\
\hline$R e$ & {$[-$} & Reynolds number, \\
\hline$S c$ & {$[-$} & Schmidt number, \\
\hline$T$ & {$\left[{ }^{\circ} \mathrm{C}\right.$ or $\left.\mathrm{K}\right]$} & dimensional temperature of the fluid, \\
\hline$T_{w}$ & {$\left[{ }^{\circ} \mathrm{C}\right.$ or $\left.\mathrm{K}\right]$} & temperature of the sheet wall, \\
\hline$T_{\infty}$ & {$\left[{ }^{\circ} \mathrm{C}\right.$ or $\left.K\right]$} & $\begin{array}{l}\text { dimensional or free stream temperature of the fluid far away from } \\
\text { the sheet wall, }\end{array}$ \\
\hline$U_{e}$ & {$[\mathrm{~m} / \mathrm{s}]$} & velocity distribution in the boundary layer for external stream, \\
\hline$u, v$ & {$[\mathrm{~m} / \mathrm{s}]$} & velocity components in the directions $x$ and $y$ \\
\hline$\hat{u}, \hat{v}$ & {$[-]$} & dimensionless velocity components in the directions $x$ and $y$, \\
\hline$x$ & {$[\mathrm{~m}]$} & axial or vertical coordinate also used as characteristic length, \\
\hline$y$ & [m] & $\begin{array}{l}\text { transverse/horizontal coordinate or non- dimensional distance to } \\
\text { the surface. }\end{array}$ \\
\hline
\end{tabular}

\section{Greek Symbols}

$\begin{array}{lll}\alpha & {\left[\mathrm{m}^{2} / \mathrm{s}\right]} & \text { thermal diffusivity, } \\ \theta & {[-]} & \text { dimensionless temperature, } \\ \kappa & {\left[\mathrm{kgm} /{ }^{\circ} \mathrm{Cs}^{3}\right]} & \begin{array}{l}\text { constant thermal conductivity, } \\ \text { heat source/sink parameter, }\end{array} \\ \lambda & {[-]} & \text { dynamic viscosity, } \\ \mu & {[\mathrm{kg} / \mathrm{ms}]} & \text { kinematic fluid viscosity, } \\ \nu & {\left[\mathrm{m}^{2} / \mathrm{s}\right]} & \text { fluid density, } \\ \rho & {\left[\mathrm{kg} / \mathrm{m}^{3}\right]} & \text { electrical conductivity of fluid, } \\ \sigma & {\left[\mathrm{ohm}^{-1} \mathrm{~m}^{-1}\right]} & \text { chemical reaction parameter, } \\ \tau & {[-]} & \text { dimensionless concentration. }\end{array}$

\section{Subscripts and Superscripts}

$j \quad[-]$ streamwise pressure gradient parameter,

$n \quad[-]$ order of reaction,

$w \quad[-]$ condition at the wall.

\section{INTRODUCTION}

The study of heat and mass transfer between a moving surface and a fluid dates back to the 1960s when Sakiadis [1, 2] published his first article and pioneering work on boundary layer flow on a continuous moving surface. This area of fluid dynamics has continued to receive attention of many researchers due to its wide applications in many engineering and geophysical applications such as geothermal reservoirs, drying of porous solids, thermal insulation, enhanced oil recovery, packed-bed catalytic reactors, cooling of nuclear reactors and underground energy transport [3, 4, 5. Ravikumar [6] investigated the heat and mass transfer effect on MHD flow of viscous fluid 
through non-homogeneous porous medium in the presence of temperature dependent heat source. The equations governing the flow are solved by a simple perturbation technique and numerical evaluation of the analytical result is reported. As expected, increasing the magnetic parameter reduces the primary profiles and the secondary velocity profiles.

Kandasamy et al. [7] studied the effects of chemical reaction, heat and mass transfer on boundary layer flow over a wedge with heat radiation in the presence of suction or injection using the Runge-Kutta Gill method. The result shows that in the presence of heat radiation the velocity and temperature of the fluid decreases and concentration of the fluid increases with increase in chemical reaction parameter. Furthermore, the velocity profiles show that the velocity increases near the plate and thereafter remains uniform. In both cases of suction and injection, it was seen that skin friction increases as chemical reaction increases. Devi and Kandasamy [8] studied the effects of heat and mass transfer on nonlinear boundary layer flow over a wedge with suction or injection. An approximate numerical solution is obtained using the Gill method. The result obtained shows that the flow field is influenced appreciably by the magnetic field and suction or injection at the wall of the wedge.

Muthucumaraswamy [9] investigated the effects of heat and mass transfer on a continuously moving isothermal vertical surface with uniform suction taking into account the homogeneous chemical reaction of first order. A theoretical solution of the problem is obtained in terms of exponential functions. It is observed that the velocity increases during the generative reaction and decreases in the destructive reaction while the concentration increase in the presence of the generative reaction. Sharma [10] discussed in detail the effect of variable thermal conductivity and heat source and sink on MHD flow near a stagnation point on a linearly stretching sheet. The numerical solution shows that the rate of heat transfer at the sheet increases due to the increase in the thermal conductivity parameter, while it decreases due to increase in stretching parameter in the absence of magnetic field and volumetric rate of heat source/sink parameter.

Devi et al. [11 analyzed a steady MHD boundary layer flow due to an exponentially stretching sheet with radiation taking into account heat source/sink. By using a fourth order Runge-Kutta method along with shooting technique, they obtained a numerical solution which shows that the momentum boundary layer thickness decreases while both thermal and concentration boundary layer thicknesses increase with an increase in the magnetic field intensity and the radiation reduces the temperature. Gangadhar and Bhaskar [12] analyzed the problem of chemically reacting MHD boundary layer flow of heat and mass transfer over a moving vertical plate in a porous medium with suction. The heat source/sink effects in thermal convection are significant where high temperature differences exist between the surface (e.g. space craft body) and the ambient fluid.

Yih [13] presented an analysis of the forced convection boundary layer flow over a wedge with uniform suction/blowing. The non-similar equations are solved using an implicit finite difference method. It was found that both suction and blowing lead to 
a decrease in the values of the local skin friction coefficient and the Nusselt number. Watanabe [14 investigated the behavior of the boundary layer over a wedge with suction or injection in forced flow. The boundary layer equations along a wedge are transformed from partial differential equations into the ordinary differential equations and the non-similar solution are obtained by means of the difference method. The solutions of the resulting equations are expressed in the form of integral equations which are in turn solved by iterative numerical quadratures. Watanabe 14 also reported that "increasing the suction/injection parameter results in wider velocity distribution and narrower temperature distribution, while decreasing the pressure gradient parameter broadens both distributions".

Bhattacharyya 15] analyzed the effect of heat source/sink on MHD flow and heat transfer over a shrinking sheet with mass suction. Employing the finite difference method using quasilinearization technique, it was found that velocity inside the boundary layer increases with increase of wall mass suction and magnetic field and accordingly the thickness of the momentum boundary layer decreases. The temperature decreases with Hartmann number, Prandtl number and heat sink parameter and the temperature increases with heat source parameter. Furthermore, for a strong heat source, heat absorption at the sheet occurs.

Ajadi [16] studied the isothermal flow of a dusty viscous incompressible conducting fluid between two types of motion in the boundary - oscillatory and non-oscillatory, under the influence of gravitational force. Within the framework of some physically realistic approximations and suitable boundary conditions, closed form solutions were obtained. It was observed that the velocity profile of the fluid decreases with increasing time and the particles respond faster to changes in the magnetic field and gravity than in the fluid. Also the skin friction decreases with increasing magnetic field. Dulal et al. 17] analyzed the influence of temperature-dependent viscosity and thermal radiation on MHD forced convection over a non-isothermal wedge. A transformed set of non-similar equations was obtained and solved by the Runge-Kutta-Fehlberg Scheme with shooting technique. The velocity and local skin friction coefficient increase with pressure gradient and magnetic field. The temperature profile increases with increasing time-dependent viscosity parameter for liquids while the temperature is higher in the case of gas viscosity parameter is positive, than for liquid viscosity parameter is negative for all values of thermal radiation parameter.

Baoheng [18] presented approximate analytical solution to Falkner-Skan wedge flow with a permeable wall of uniform suction. Comparisons of results from the Homotopy Analysis Method are made with the numerical method by 4th-order Runge-Kutta method combined with Newton-Raphson technique which established validity of the results. It is observed that the velocity profile increases with increasing suction parameter and decreases with increasing pressure gradient parameter.

This present work is undertaken to study the behavior of velocity, temperature and concentration profiles in the presence of a heat source and chemical reaction using the Homotopy Perturbation Method (HPM). The approximate analytical solutions obtained are further analyzed using MAPLE 17 symbolic package. Analysis involving 
other important parameters such as Schmidt number, Prandtl number and Hartmann is carried out.

\section{Formulation of the Problem}

Let us consider a steady, the non-isothermal, unsteady, two-dimensional flow of an electrically conducting fluid with a reaction source based on the one-step exothermic reaction mechanism of the form:

$$
F+O \longrightarrow \text { Product }+ \text { Energy },
$$

where $F$ is the fuel and $O$ is the oxidizer. The boundary layer is assumed flat, while the reaction source is placed in the viscous region as shown in Figure 1

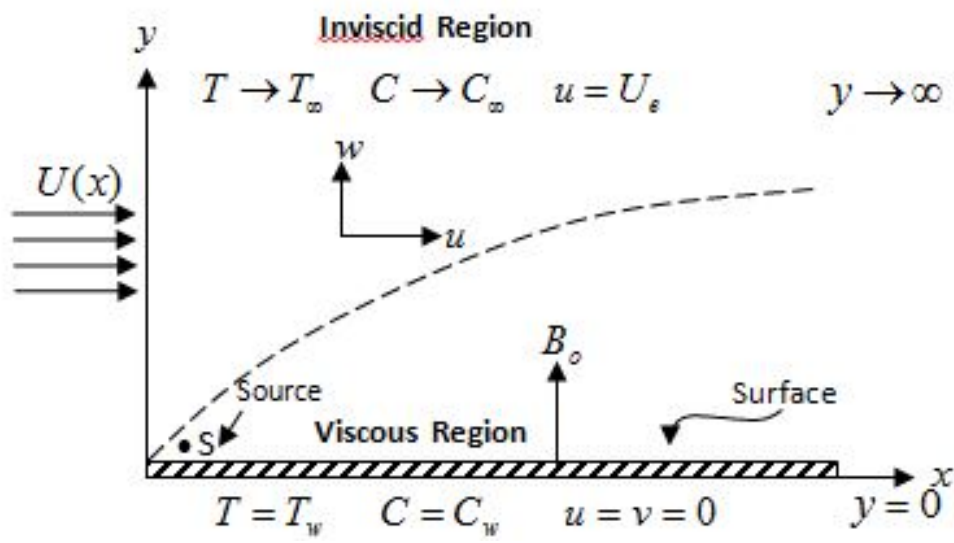

Figure 1. Schematic diagram of the flow

The governing equations of the MHD boundary layer flow in the presence of uniform transverse magnetic field in the viscous region are

$$
\begin{gathered}
\frac{\partial u}{\partial x}+\frac{\partial v}{\partial y}=0 \\
u \frac{\partial u}{\partial x}+v \frac{\partial u}{\partial y}=\nu \frac{\partial^{2} u}{\partial y^{2}}-\frac{\sigma B_{0}^{2}}{\rho} u-\frac{1}{\rho} \frac{\partial P}{\partial x} \\
u \frac{\partial T}{\partial x}+v \frac{\partial T}{\partial y}=\frac{\kappa}{\rho C_{p}} \frac{\partial^{2} T}{\partial y^{2}}+\frac{Q_{o}}{\rho C_{p}}\left(T-T_{\infty}\right)+\frac{Q_{o}}{\rho C_{p}} C^{n} e^{-\frac{E}{R T}} \\
u \frac{\partial C}{\partial x}+v \frac{\partial C}{\partial y}=\frac{D}{\rho C_{p}} \frac{\partial^{2} C}{\partial y^{2}}+\delta C^{n} e^{-\frac{E}{R T}} .
\end{gathered}
$$

The boundary conditions for the velocity components, temperature and concentration are given by:

$$
u=0, \quad v=-v_{w}, \quad C=C_{w}, \quad T=T_{w} \quad \text { at } \quad y=0,
$$




$$
u=U_{e}=a x^{m}, \quad C \rightarrow C_{\infty}, \quad T \rightarrow T_{\infty} \quad \text { as } \quad y \rightarrow \infty .
$$

where, $u, v, U_{e}, v_{w}>0$ are the $x$-component of velocity, $y$-component of velocity, free stream velocity and prescribed distribution of wall mass suction through the porous plate, respectively.

Introducing the stream function $\psi$, the velocity components $u$ and $v$ can be written as:

$$
u=\frac{\partial \psi}{\partial y} \quad \text { and } \quad v=-\frac{\partial \psi}{\partial x} .
$$

We now introduce the following dimensionless variables:

$$
\begin{array}{rlrl}
\psi=\sqrt{\nu a} x^{\frac{m+1}{2}} & f(\eta), & \eta & =\frac{y}{x} \sqrt{R e}=\sqrt{\frac{a}{\nu}} y x^{\frac{m-1}{2}}, \\
U_{e}=a x^{m}, & \theta=\frac{T-T_{\infty}}{T_{w}-T_{\infty}}, & \phi
\end{array}
$$

The transformed momentum equation 3.2 becomes

$$
\begin{gathered}
f^{\prime \prime \prime}+\frac{m+1}{2} f f^{\prime \prime}+m\left[1-\left(f^{\prime}\right)^{2}\right]-M^{2} f^{\prime}=0, \\
f(0)=S, f^{\prime}(0)=0, f^{\prime}(\infty)=1 .
\end{gathered}
$$

The energy equation 3.3 reduces to

$$
\begin{gathered}
\theta^{\prime \prime}+\operatorname{Pr}\left(\frac{m+1}{2} f \theta^{\prime}+\lambda \theta+\tau \phi e^{\theta}\right)=0, \\
\theta(0)=1, \theta(\infty)=0,
\end{gathered}
$$

and the specie equation 3.4 gives

$$
\begin{gathered}
\phi^{\prime \prime}+S c\left(\frac{m+1}{2} f \phi^{\prime}-\tau \operatorname{Re} \phi e^{\theta}\right)=0, \\
\phi(0)=1, \phi(\infty)=0,
\end{gathered}
$$

where primes denote the differentiation with respect to $\eta$ and $M=\left(\frac{\sigma B_{0}^{2}}{\rho a}\right)^{\frac{1}{2}}, R e=\frac{x U_{e}}{\nu}=\frac{a x^{m+1}}{\nu}, \operatorname{Pr}=\frac{\mu C_{p}}{\kappa}, S c=\frac{\nu}{D}, \lambda=\frac{Q_{o}}{\rho C_{p} a}, \tau=\frac{\delta \nu}{U^{2}}$ and $S=\frac{v_{w}}{(a \nu)^{1 / 2}}>0$ is the mass suction parameter.

\section{Mathematical Procedure and Solution}

The Homotopy Perturbation Method (HPM) is based on the concept of topology, which has been discovered to be an effective and efficient tool for solving non-linear equations [19-21]. To illustrate Homotopy Perturbation Method, we consider the nonlinear equation:

$$
A(u)-f(r)=0, r \in \Omega,
$$

with the boundary conditions:

$$
B\left(u, \frac{d u}{d n}\right)=0, r \in \Gamma,
$$


where $A$ is a general differential operator, $B$ is a boundary operator, $f(r)$ is a known analytic function, and $\Gamma$ is the boundary of domain $\Omega$. The operator $A$ are generally divided into two parts; $L$ and $N$, where, $L$ and $N$ are linear and nonlinear parts of $A$ respectively. Therefore, equation (4.1) may be written as

$$
L(u)+N(u)-f(r)=0 .
$$

We construct a Homotopy $v(r, p): \Omega \times[0,1] \rightarrow \Re$

$$
H(v, p)=\left[L(v)-L\left(u_{0}\right)\right]+p L\left(u_{0}\right)+p[N(u)-f(r)]=0,
$$

or

$$
H(v, p)=\left[L(v)-L\left(u_{0}\right)\right]+p[A(u)-f(r)]=0 .
$$

Where, $p \epsilon[0,1]$ is called the homotopy parameter and $u_{0}$ is an initial approximation of equation 4.3 . At the two extremes $p=0$ and $p=1$, we have

$$
H(v, 0)=\left[L(v)-L\left(u_{0}\right)\right]=0 \quad \text { and } \quad H(v, 1)=[A(u)-f(r)] .
$$

In the interval $0<p<1$, the homotopy $H(v, p)$ deforms from $L(v)-L\left(u_{0}\right)$ to $A(u)-f(r)$. Thus, the solution of equtions (3.9), (3.11) and 3.13) may be expressed as

$$
v=v_{0}+v_{1} p+v_{2} p^{2}+v_{3} p^{3}+v_{4} p^{4}+\ldots
$$

Eventually, at $p=1$, the system takes the original form of the equation and the final stage of deformation gives the desired solution. Thus taking limits

$$
u=\lim _{p \rightarrow 1} v=v_{0}+v_{1}+v_{2}+v_{3}+v_{4}+\ldots
$$

We start by applying the Homotopy Perturbation Method to equations 3.9 to 3.14 and we define the homotopy as

$$
\begin{gathered}
f^{\prime \prime \prime}-f_{0}^{\prime \prime \prime}+p f_{0}^{\prime \prime \prime}+p\left(\frac{m+1}{2} f f^{\prime \prime}+m\left[1-\left(f^{\prime}\right)^{2}\right]-M^{2} f^{\prime}\right)=0, \\
\theta^{\prime \prime}-\theta_{0}^{\prime \prime}+p \theta_{0}^{\prime \prime}+p\left[\operatorname{Pr}\left(\frac{m+1}{2} f \theta^{\prime}+\lambda \theta+\tau \phi e^{\theta}\right)\right]=0, \\
\phi^{\prime \prime}-\phi_{0}^{\prime \prime}+p \phi_{0}^{\prime \prime}+p\left[S c\left(\frac{m+1}{2} f \phi^{\prime}-\tau \operatorname{Re} \phi e^{\theta}\right)\right]=0 .
\end{gathered}
$$

Suppose that the solutions of $f, \theta$ and $\phi$ take the form

$$
\begin{gathered}
f(\eta)=f_{0}(\eta)+f_{1}(\eta) p+f_{2}(\eta) p^{2}+f_{3}(\eta) p^{3}+f_{4}(\eta) p^{4}+\ldots \\
\theta(\eta)=\theta_{0}(\eta)+\theta_{1}(\eta) p+\theta_{2}(\eta) p^{2}+\theta_{3}(\eta) p^{3}+\theta_{4}(\eta) p^{4}+\ldots \\
\phi(\eta)=\phi_{0}(\eta)+\phi_{1}(\eta) p+\phi_{2}(\eta) p^{2}+\phi_{3}(\eta) p^{3}+\phi_{4}(\eta) p^{4}+\ldots,
\end{gathered}
$$

substituting equation (4.12) into equation 4.9 and picking terms in order of $p$, we have

$$
\begin{gathered}
p^{0}: \quad f_{0}(\eta)-f_{0}(\eta)=0, \\
\left.p^{1}: \quad f_{1}^{\prime \prime \prime}+f_{0}^{\prime \prime \prime}+\frac{m+1}{2} f_{0} f_{0}^{\prime \prime}+m\left[1-\left(f_{0}^{\prime}\right)^{2}\right]-M^{2} f_{0}^{\prime}\right)=0, \\
\left.p^{2}: \quad f_{2}^{\prime \prime \prime}+\frac{m+1}{2}\left(f_{0} f_{1}^{\prime \prime}+f_{1} f_{0}^{\prime \prime}\right)-m\left(2 f_{0}^{\prime} f_{1}^{\prime}\right)-M^{2} f_{1}^{\prime}\right)=0, \\
f_{0}(0)=0, f_{0}^{\prime}(0)=0, f_{0}^{\prime}(\infty)=0 .
\end{gathered}
$$


From the momentum equation and the boundary conditions, we take our initial guess to be

$$
f_{0}(\eta)=\eta-\left(1-e^{\eta}\right)
$$

Solving equation 4.16 we get

$$
\begin{gathered}
f_{1}^{\prime \prime \prime}=e^{\eta}-\frac{m+1}{2}\left(\eta-\left(1-e^{\eta}\right)\right) e^{\eta}+m\left[1-\left(1-e^{\eta}\right)^{2}\right]+M^{2}\left(\eta-\left(1-e^{\eta}\right)\right)=0 \\
f_{1}=-\frac{1}{16} e^{-2 \eta} m+\frac{1}{2} e^{-\eta} m+3 m e^{\eta}+\frac{1}{16} e^{-2 \eta}+\frac{1}{2} e^{-\eta} \eta+M^{2} e^{-\eta} \\
+\frac{1}{6} M^{2} \eta^{3}+\frac{1}{2} D_{1} \eta^{2}+D_{2} \eta+D_{3}
\end{gathered}
$$

Using the boundary condition, we have

$$
\begin{gathered}
f_{1}=-\frac{1}{16} e^{-2 \eta} m+\frac{1}{2} e^{-\eta} m+3 m e^{\eta}+\frac{1}{16} e^{-2 \eta}+\frac{1}{2} e^{-\eta} \eta+M^{2} e^{-\eta}+\frac{1}{6} M^{2} \eta^{3} \\
+\frac{1}{2}\left(\frac{1}{4} M^{2} e^{-4}-\frac{9}{4} M^{2}-\frac{1}{32} e^{-8} m+\frac{9}{8} e^{-4} m+\frac{1}{32} e^{-8}+\frac{3}{8} e^{-4}\right. \\
\left.+\frac{3}{32}-\frac{19}{32} m\right) \eta^{2}+\left(M^{2}+\frac{19}{8} m-\frac{3}{8}\right) \eta-M^{2}-\frac{47}{16} m-\frac{1}{16} .
\end{gathered}
$$

From equation 4.17

$$
\left.f_{2}^{\prime \prime \prime}=-\frac{m+1}{2}\left(f_{0} f_{1}^{\prime \prime}+f_{1} f_{0}^{\prime \prime}\right)+m\left(2 f_{0}^{\prime} f_{1}^{\prime}\right)+M^{2} f_{1}^{\prime}\right)
$$

The solutions of $f_{2}, f_{3}$ and $f_{4}$ have been obtained by integration using MAPLE 17 symbolic package. Hence, the fourth order approximation is given by

$$
f=f_{0}+f_{1}+f_{2}+f_{3}+f_{4}+\ldots
$$

Similarly, substituting equations 4.12 and 4.13 into equation 4.10 and picking terms in order of $p$, we have

$$
\begin{gathered}
p^{0}: \quad \theta_{0}^{\prime \prime}(\eta)-\theta_{0}^{\prime \prime}(\eta)=0, \\
p^{1}: \quad \theta_{1}^{\prime \prime}+\theta_{0}^{\prime \prime}+\operatorname{Pr}\left(\frac{m+1}{2} f_{0} \theta_{0}^{\prime}+\lambda \theta_{0}+\tau \theta_{0} e^{\theta_{0}}\right)=0, \\
p^{2}: \quad \theta_{2}^{\prime \prime}+\operatorname{Pr}\left(\frac{m+1}{2}\left(f_{0} \theta_{1}^{\prime}+f_{1} \theta_{0}^{\prime}\right)+\lambda \theta_{1}+\tau \phi_{1} e^{\theta_{0}}\right)=0, \\
\theta_{0}(0)=1, \theta_{0}(\infty)=0 .
\end{gathered}
$$

Similarly, from the boundary conditions we take our initial guess to be

$$
\theta_{0}(\eta)=e^{\eta}
$$

Solving equation 4.25

$$
\theta_{1}^{\prime \prime}=-\theta_{0}^{\prime \prime}-\operatorname{Pr}\left(\frac{m+1}{2} f_{0} \theta_{0}^{\prime}-\lambda \theta_{0}-\tau \theta_{0} e^{\theta_{0}}\right) .
$$


Similarly, substituting (4.18) and (4.27), then by integration, we obtain

$$
\begin{aligned}
\theta_{1}=-\frac{1}{2} \text { Pr }_{m} e^{-\eta}-\frac{1}{2} \text { Prme }^{-\eta}-\frac{1}{2} \text { Pr } e^{-\eta}-\frac{1}{2} \operatorname{Pre}^{-\eta}-\lambda e^{-\eta} \\
\quad-\tau e^{-\eta}-\frac{1}{8}{\text { Pr } \eta m e^{-2 \eta}}^{-e^{-\eta}}-\frac{1}{8} \operatorname{Pr}^{-2 \eta}-\frac{1}{4} e^{-2 \eta} \tau+E_{1} \eta+E_{2} .
\end{aligned}
$$

Using the boundary conditions we have

$$
\begin{aligned}
& \theta_{1}=-\frac{1}{2} \operatorname{Pr}_{m} e^{-\eta}-\frac{1}{2} \operatorname{Prme}^{-\eta}-\frac{1}{2} \operatorname{Pr}^{-\eta}- \frac{1}{2} \operatorname{Pre}^{-\eta}-\lambda e^{-\eta}-\tau e^{-\eta} \\
&-\frac{1}{8} \operatorname{Pr}^{-\eta} e^{-2 \eta}-e^{-\eta}-\frac{1}{8} \operatorname{Pr}^{-2 \eta}-\frac{1}{4} e^{-2 \eta} \tau+\left(\frac{5}{8} \operatorname{Prme}^{-4}+\frac{1}{32} m e^{-8} \operatorname{Pr}+\frac{5}{8} e^{-4} \operatorname{Pr}\right. \\
&\left.+\frac{1}{4} \lambda e^{-4}+\frac{1}{4} \tau e^{-4}+\frac{1}{32} e^{-8} \operatorname{Pr}+\frac{1}{16} \tau e^{-8}+\frac{1}{4} e^{-4}-\frac{1}{4}-\frac{5}{32} \operatorname{Pr} m-\frac{5}{32} \operatorname{Pr}-\frac{1}{4} \lambda-\frac{5}{16} \tau\right) \eta \\
&+\frac{5}{8} \operatorname{Pr} m+\frac{5}{8} \operatorname{Pr}+\lambda+\frac{5}{4} \tau+1 .
\end{aligned}
$$

From equation 4.26

$$
\theta_{2}^{\prime \prime}=-\operatorname{Pr}\left(\frac{m+1}{2}\left(f_{0} \theta_{1}^{\prime}+f_{1} \theta_{0}^{\prime}\right)+\lambda \theta_{1}+\tau \phi_{1} e^{\theta_{0}}\right) .
$$

Similarly, the solutions of $\theta_{2}, \theta_{3}$ and $\theta_{4}$ are obtained but have not been written here for space economy. The fourth order approximation is given by

$$
\theta=\theta_{0}+\theta_{1}+\theta_{2}+\theta_{3}+\theta_{4}+\ldots
$$

Similarly, substituting 4.12 -4.14 in 4.11) and picking terms in order of $p$, we have

$$
\begin{gathered}
p^{0}: \quad \phi_{0}^{\prime \prime}(\eta)-\phi_{0}^{\prime \prime}(\eta)=0 \\
p^{1}: \quad \phi_{1}^{\prime \prime}+\phi_{0}^{\prime \prime}+\left(\frac{m+1}{2} S c f_{0} \phi_{0}^{\prime}-\tau S c R e \phi_{0} e^{\theta_{0}}\right)=0 \\
p^{2}: \quad \phi_{2}^{\prime \prime}+\left(\frac{m+1}{2} S c\left(f_{0} \theta_{1}^{\prime}+f_{1} \theta_{0}^{\prime}\right)-\tau S c R e \phi_{1} e^{\theta_{0}}\right)=0 \\
\phi(0)=1, \phi(\infty)=0 .
\end{gathered}
$$

Similarly, from the boundary conditions we take our initial guess to be

$$
\phi_{0}(\eta)=e^{\eta}
$$

Similarly, solutions of $\phi_{1}, \phi_{2}, \phi_{3}$ and $\phi_{4}$ are obtained but have not been written here for space economy. The fourth order approximation is given by

$$
\phi=\phi_{0}+\phi_{1}+\phi_{2}+\phi_{3}+\phi_{4}+\ldots
$$




\section{Results And Discussion.}

In our computation, the following values have been selected, Prandtl number $(\operatorname{Pr})$ is taken to be 0.72 which corresponds to air and the values of Schmidt number $(S c)$ is chosen as 0.62 and 0.78 representing diffusing chemical species of $\mathrm{H}_{2} \mathrm{O}$ and $\mathrm{NH}_{3}$, respectively at $25^{\circ} \mathrm{C}$ and $1 \mathrm{~atm}$, while $m=0.1, M=0.2, \lambda=0.01, R e=1$ and $\tau=0.2$, unless stated otherwise.

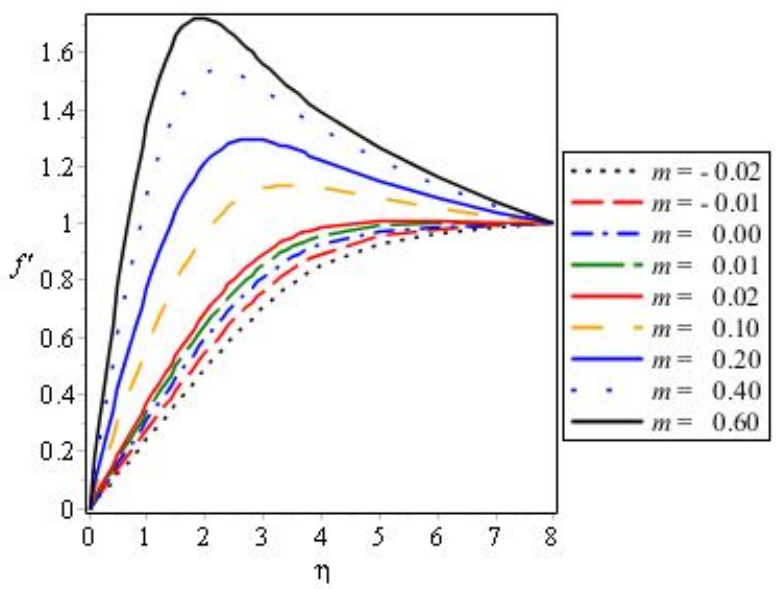

Figure 2. Graphs of $f^{\prime}$ vs $\eta$ for some $m$

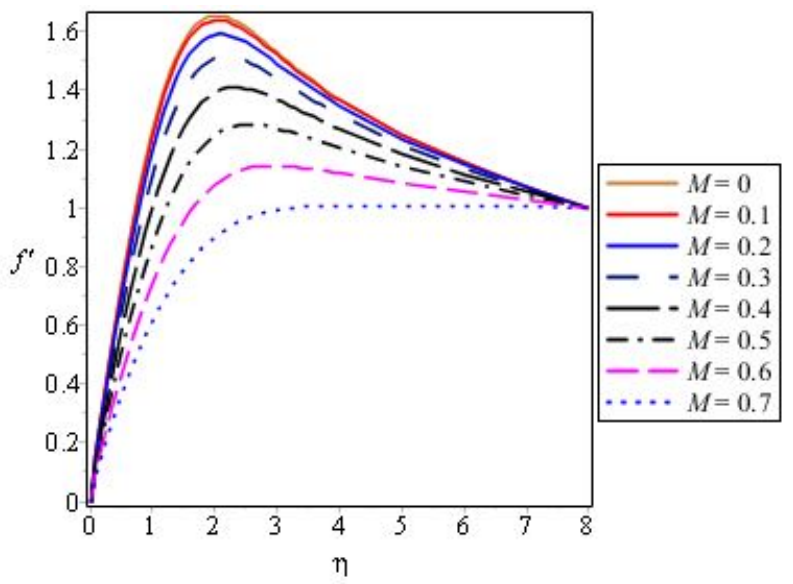

Figure 3. Graphs of $f^{\prime}$ vs $\eta$ for some $M$

Graphical results for the velocity profiles are displayed in Figures 2 and 3 for the influence of $m$ and $M$, respectively. 


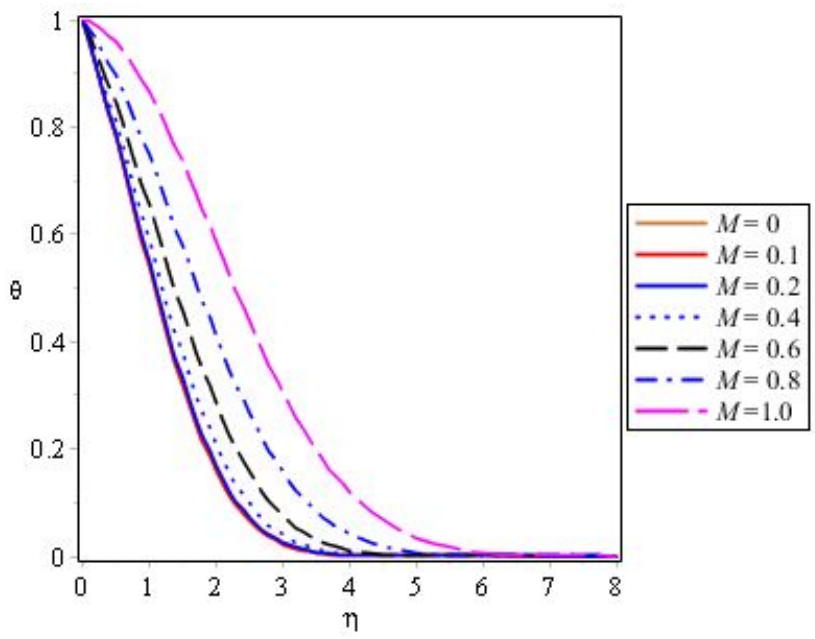

Figure 4. Graphs of $\theta$ vs $\eta$ for some $M$

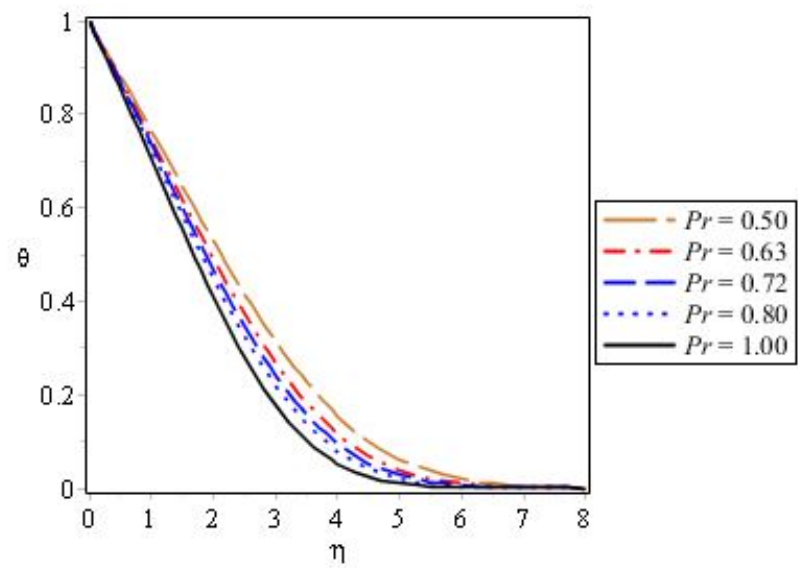

Figure 5. Graphs of $\theta$ vs $\eta$ for some $\operatorname{Pr}$ without chemical reaction

It is observed in Figure 2 that the velocity profiles for $m>0$ is higher than $m<0$, noting that the velocity profiles is convex for $m>0$. This is because for a positive value of $m$, pressure gradient is negative and for a negative value of $m$, pressure gradient is positive $\left(-\frac{1}{\rho} \frac{\partial P}{\partial x}=U_{e} \frac{d U_{e}}{d x}=m\right)$. A negative pressure gradient occurs as a result of pressure decreases in the direction of fluid flow across the boundary layer. Thus the fluid within the boundary layer has enough momentum to overcome the resistance which is trying to push it backward and the flow accelerates. For a positive pressure gradient the pressure increases in the direction of flow, the fluid within the 


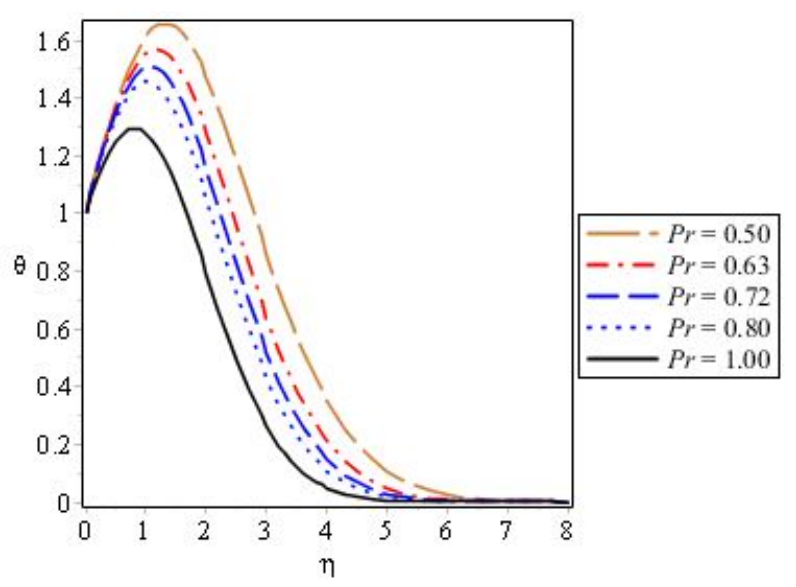

Figure 6. Graphs of $\theta$ vs $\eta$ for some $\operatorname{Pr}$ with chemical reaction

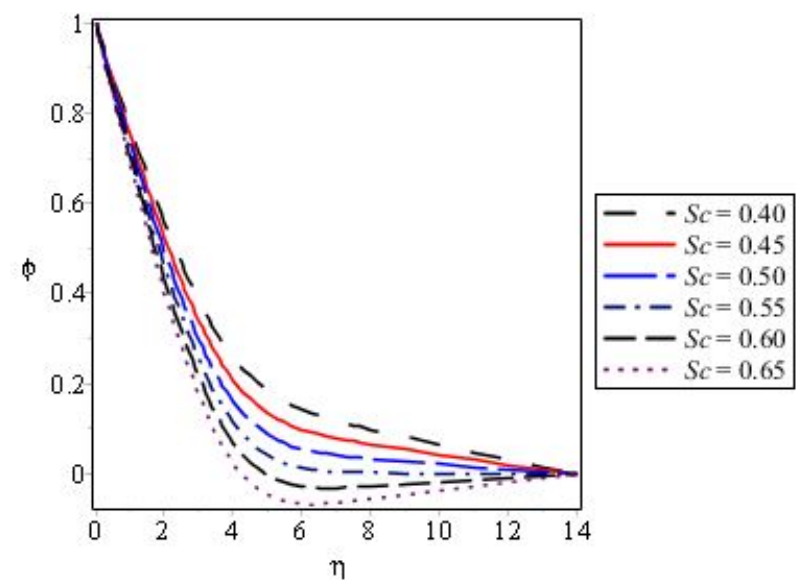

Figure 7. Graphs of $\phi$ vs $\eta$ for some $S c$ at $M=0.5$

boundary layer has little momentum to overcome this resistance which could make the flow retarded and possibly lead to flow reversal.

The impacts of the Hartmann number $M$ on the velocity and temperature profiles are very significant from a practical point of view. In Figures 3 and 4 the variations in velocity field and temperature distribution for several values of $M$ are presented. The dimensionless velocity $f^{\prime}(\eta)$ decreases with increasing values of $M$. This is because the application of transverse magnetic field will result in a resistive force (Lorentz force) similar to drag force which tends to resist the fluid flow thus reducing its velocity, as established by [6] and [8]. On the other hand, Figure 4 shows that the temperature 


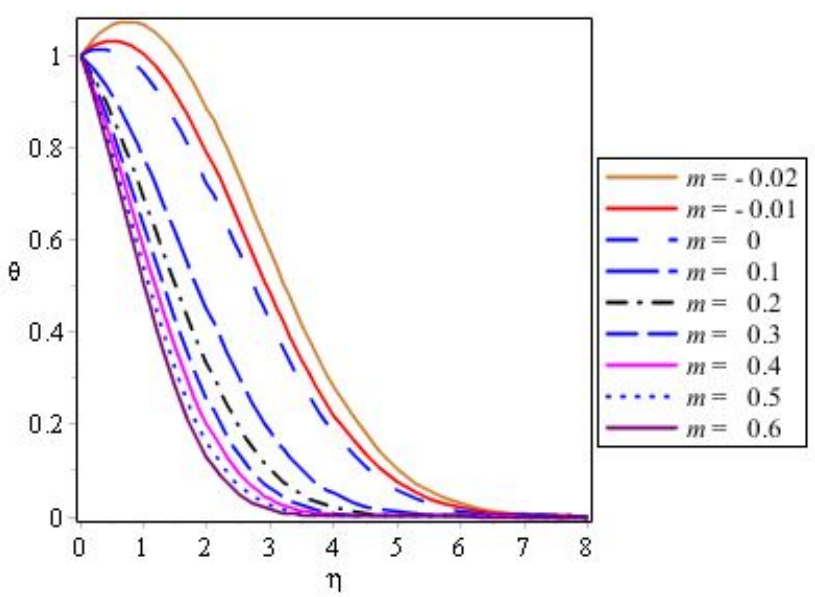

Figure 8. Graphs of $\theta$ vs $\eta$ for some $m$

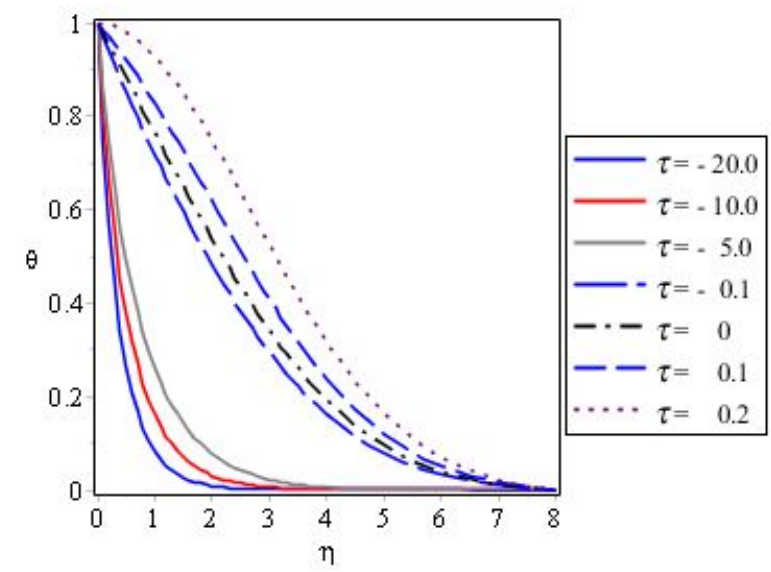

Figure 9. Graphs of $\theta$ vs $\eta$ for some $\tau$

profiles $\theta(\eta)$ increases with increase in $M$ due to the same reason. Similar behavior is observed in Figure 10 for concentration profiles $\phi(\eta)$.

The effect of Prandtl number on the temperature profile in the absence of chemical reaction is shown in Figure 5. It is observed that an increase in the Prandtl number $(\mathrm{Pr})$ decreases the temperature profile. This is justified in that higher values of Prandtl number are equivalent to decrease in the thermal conductivity of the fluid and therefore heat flow is reduced. Hence, there is a reduction in temperature. Figure 6] shows the similar temperature profile pattern with increasing Prandtl number as 


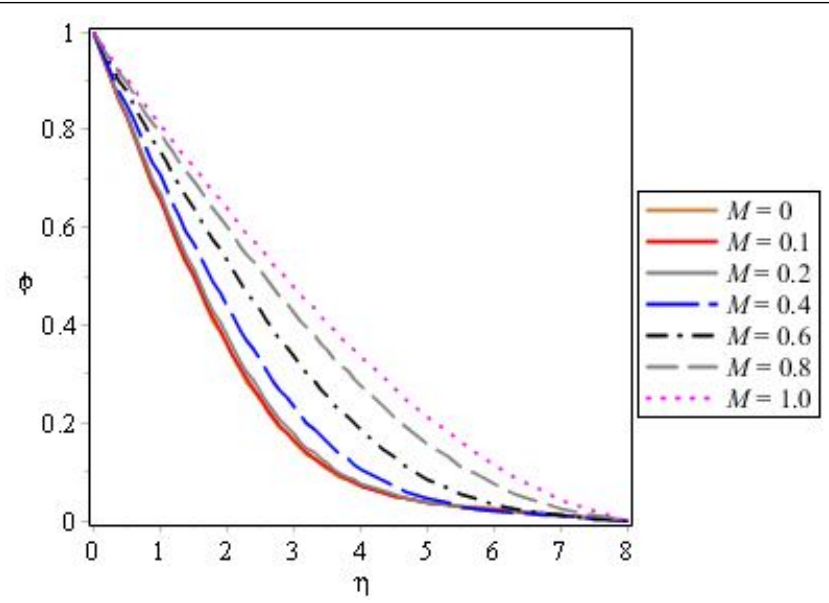

Figure 10. Graphs of $\phi$ vs $\eta$ for some $M$

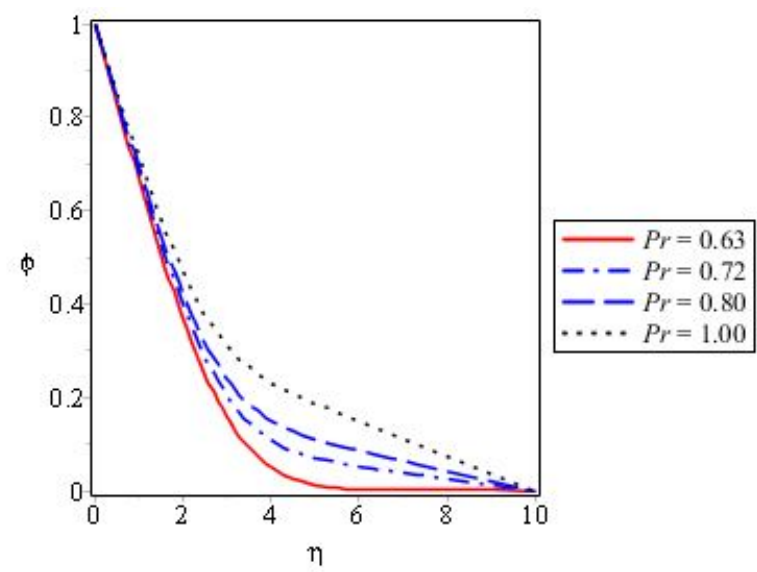

Figure 11. Graphs of $\phi$ vs $\eta$ for some $\operatorname{Pr}$

seen in [6], except for the temperature peaks in the interval $0<\eta<1.5$, which may be regarded as the reaction zone - a point where the reactant is completely consumed.

Figure 7 shows the effect of Schmidt number on the concentration profile. It is observed that higher Schmidt number reduces the concentration profile. Figure 8 shows that an increase in pressure gradient $(m)$ decreases the temperature profile, which confirms that negative pressure gradient implies accelerated flow, leading to easy convection of heat and hence decrease in temperature. 


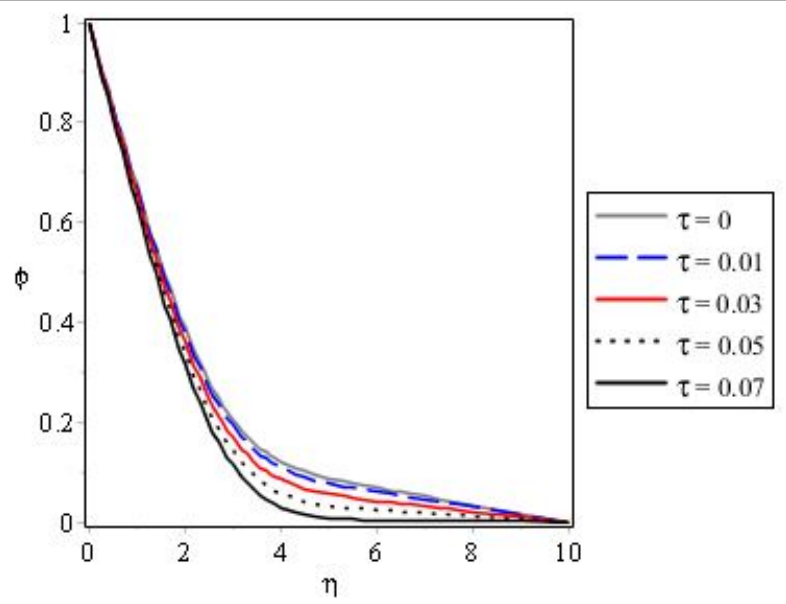

Figure 12. Graphs of $\phi$ vs $\eta$ for some $\tau$

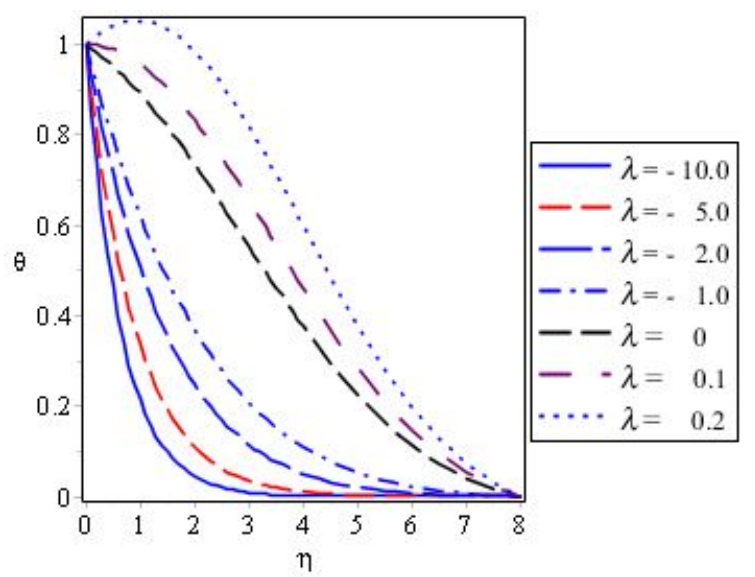

Figure 13. Graphs of $\theta$ vs $\eta$ for some $\lambda$

From Figures 9 and 12 it is clear that increasing the chemical reaction term increases the temperature profile while it decreases concentration profile, respectively which is in agreement with the result of Kandasamy [7. This is because an exothermic reaction involves the release of heat to the surroundings while depletion of the reactant is in process. Figures 7 and 11 show that concentration profile decreases and increases with Schmidt number $(S c)$ and Prandtl number $(P r)$ respectively. This is because a large Schmidt number occurs as a result of decrease in mass diffusivity, which reduces concentration across the boundary layer and in general reduces concentration of reactant within the boundary layer. 


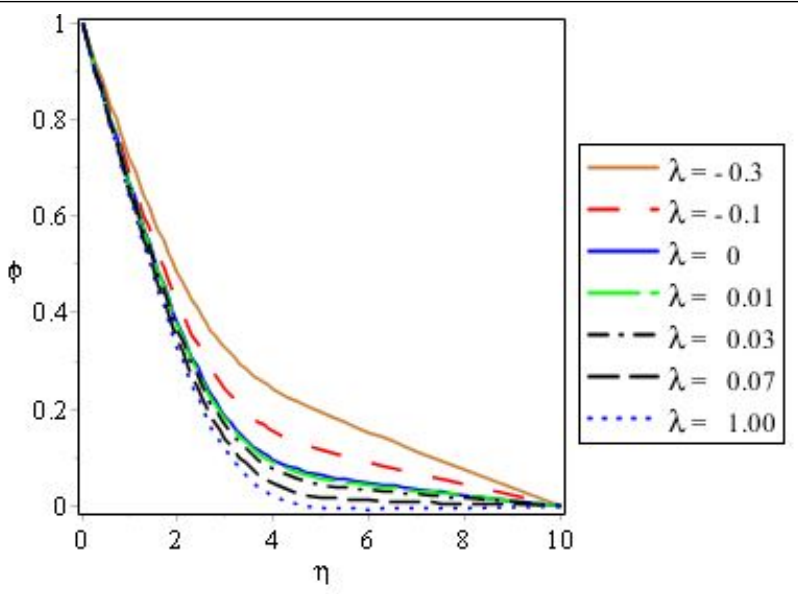

Figure 14. Graphs of $\phi$ vs $\eta$ for some $\lambda$

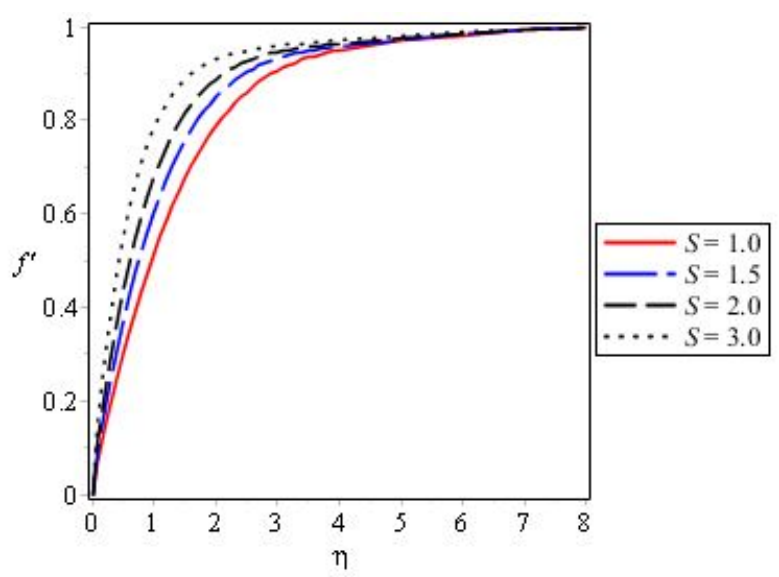

Figure 15. Suction effects on velocity profile

In Figure 11, Prandtl number $(P r)$ has a negligible effect on concentration $\phi(\eta)$. Figures 13 and 14 show the variation of heat source parameter on temperature and concentration, respectively. It is quite interesting to note that a relatively high heat source term results in a very high temperature at the reaction zone, while it decreases the concentration profile. The velocity profiles for various values of suction parameter $S$ are depicted by Figure 15 . It is noted that the velocity profiles increase as applied suction increases and this makes the momentum boundary layer thinner.

From Figure 16 it is seen that the wall suction affects the temperature distribution: increase in suction decreases the temperature, meaning that the thermal boundary 


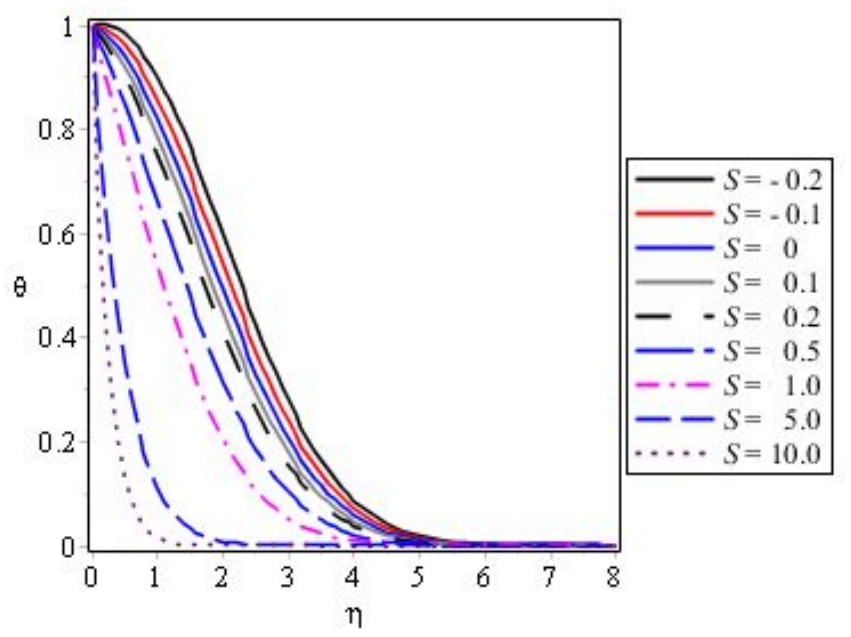

Figure 16. Suction effects on temperature profile

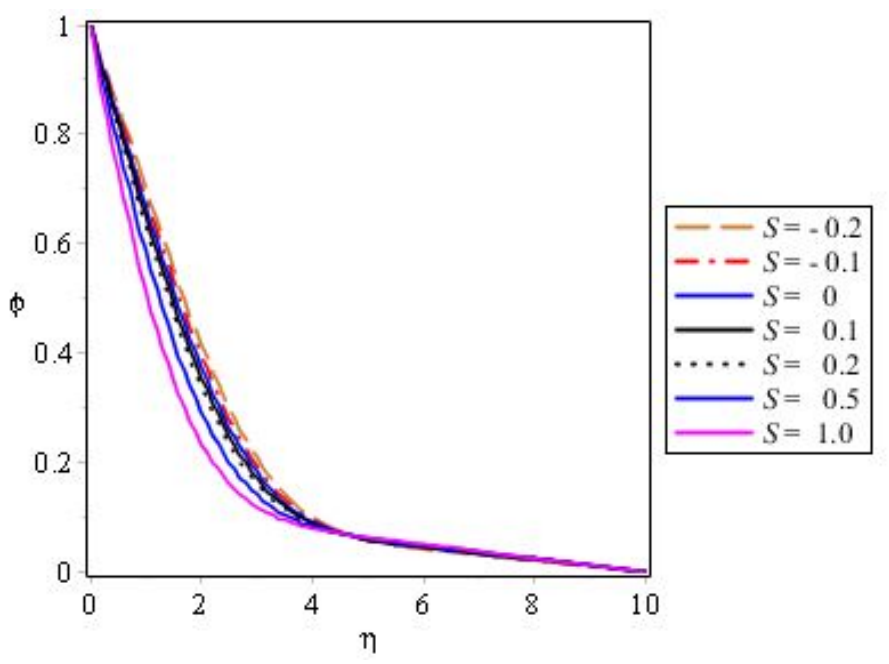

Figure 17. Suction effects on concentration profile

layer thickness reduces. Also, Figure 17] shows the suction effects on concentration profile.

The behavior of the local skin friction coefficient $\left(-f^{\prime \prime}(0)\right)$, Nusselt number $\left(\theta^{\prime}(0)\right)$ and Shearwood number $\left(-\phi^{\prime}(0)\right)$ with respect to the embedded parameters in the system were also carried out as shown in Table 1. 
Table 1. Computation showing $f^{\prime \prime}(0), \theta^{\prime}(0)$ and $\phi^{\prime}(0)$

\begin{tabular}{|c|l|l|l|l|l|l|l|l|}
\hline$m$ & $M$ & $P r$ & $S c$ & $\tau$ & $\lambda$ & $-f^{\prime \prime}(0)$ & $\theta^{\prime}(0)$ & $-\phi^{\prime}(0)$ \\
\hline 0.2 & 0.1 & 0.2 & 0.2 & 0.1 & 0.1 & 0.638110901 & -0.025503349 & 0.2440132260 \\
0.2 & 0.1 & 0.2 & 0.2 & 0.1 & 0.3 & 0.638110901 & 1.030907124 & 0.2440132260 \\
0.2 & 0.1 & 0.2 & 0.2 & 0.2 & 0.1 & 0.638110901 & 2.835536353 & 0.8162211665 \\
0.2 & 0.1 & 0.2 & 0.2 & 0.3 & 0.1 & 0.638110901 & 3.431586291 & 0.9354311542 \\
0.2 & 0.1 & 0.2 & 0.3 & 0.1 & 0.1 & 0.638110901 & -0.025503349 & 0.2916972210 \\
0.2 & 0.1 & 0.2 & 0.4 & 0.1 & 0.1 & 0.638110901 & 0.125412432 & 0.1254124320 \\
0.2 & 0.1 & 0.3 & 0.2 & 0.1 & 0.1 & 0.638110901 & 0.125412432 & 0.1254124320 \\
0.2 & 0.1 & 0.4 & 0.2 & 0.1 & 0.1 & 0.638110901 & 0.188537086 & 0.1885370865 \\
0.2 & 0.2 & 0.2 & 0.2 & 0.1 & 0.1 & 0.644682861 & 0.125412432 & 0.1254124320 \\
0.2 & 0.3 & 0.2 & 0.2 & 0.1 & 0.1 & 2.206428834 & 0.125412432 & 0.1254124320 \\
0.3 & 0.3 & 0.2 & 0.2 & 0.1 & 0.1 & 0.735052197 & 0.127516587 & 0.1275165872 \\
\hline
\end{tabular}

From the table it can be seen that the skin friction and the Nusselt number increase with increasing pressure gradient $m$ while the Shearwood number decreases. The heat source parameter has no significant effect on local skin friction coefficient and Shearwood number but increases Nusselt number. Also the local skin friction coefficient is not affected or influenced by the chemical reaction parameter, Prandtl number or Schmidt number; Nusselt number increases with increasing chemical reaction parameter, Prandtl number and Schmidt number; Sherwood number increases with increasing chemical reaction parameter and Prandtl number but decreases with increasing Schmidt number. Furthermore the Nusselt and Sherwood numbers are not influenced in any way by Hartmann number $M$ but the skin friction coefficient increases with increasing $M$. This is because Hartmann number $M$ represents the relative influence of the magnetohydrodynamic drag force (Lorentz body force) and the momentum force on the flow.

\section{Conclusion}

This present study investigated the behavior of MHD flow and heat transfer in the presence of heat source and chemical reaction over a stretching sheet. The governing continuity, momentum, energy and concentration equations of the flow are non-dimensionalized by introducing some standard dimensionless variables. The resulting equations were solved by employing Homotopy Perturbation Method (HPM). The approximate analytical solutions obtained are further analyzed using MAPLE 17 symbolic package. Analyses involving some important parameters such as Schmidt number, Prandtl number, Hartmann number, pressure gradient, chemical reaction and heat source parameters were carried out. The results are summarized as follows

- The temperature increases while concentration decreases with increasing heat source parameter.

- The concentration profile reduces with increasing Schmidt number .

- The magnetic field and Prandtl number enhance the temperature profile.

- Increasing suction parameter increases velocity but decreases temperature. 
- The skin friction coefficient is not affected or influenced by chemical reaction parameter, Prandtl number or Schmidt number; the Nusselt number increases with increasing chemical reaction parameter, Prandtl number, Schmidt number; the Sherwood number increases with increasing chemical reaction parameter and Prandtl number but decreases with increasing Schmidt number.

Acknowledgement. This work has been supported in part by the Nigerian Tertiary Education Trust Fund (TETfund). Acknowledgment also goes to the Central European University Fellowship Programme, Budapest, where the work was initiated during the visit of the second author as a Research Fellow. Authors acknowledge with gratitude the constructive comments and suggestions of the reviewers which improved the quality of the paper.

\section{REFERENCES}

1. SAKiadis, B. C.: Boundary-layer behaviour on continous solid surface. I. Boundarylayer equations for two-dinmensional and axisymmettic flow. AIChE Journal, 7, (1961), $26-28$.

2. SAKiadis, B. C.: Boundary-layer behaviour on continous solid surface. II. Boundarylayer equations for two-dinmensional and axisymmettic flow. AIChE Journal, 7, (1961), 221-225.

3. Pijush K. Kundu and Ira M. Cohen: Fluid Mechanics. Fourth edition, Elsevier, The Boulevard, Langford lane Kidlington, Oxford, UK, 2008.

4. WANG, C. Y.: Liquid film on an unsteady stretching sheet. Quarterly of Applied Mathematics, 48(4), (1990), 601-610.

5. FAng, T. and Zhang, J.: Flow between two strehable disks - An Exact solution of the Navier-Stokes equation. International Communication in Heat and Mass Transfer, 35, (2008), 892-895.

6. Ravikumar, V., Kaju M. C. and Raju G. S. S.: Heat and mass transfer effects on MHD flow of viscous fluid through non-homogeneous porous medium in presence of temperature dependent heat source. International Journal of Contemporary Mathematical Sciences, 7(32), (2012), 1597-1604.

7. Kandasamy, R., AbD, W. B., RaJ, M. and Khamis, A. B.: Effects of chemical reaction, heat and mass transfer on boundary layer flow over a porous wedge with radiation in the presence of suction or injection. Theoretical and Applied Mechanics, Belgrade. 33(2), (2006), 123-148.

8. Devi, A. S. P. and Kandasamy, R.: Effects of heat and mass transfer on MHD laminar boundary layer flow over a wedge with suction or injection. Journal of Energy Heat and Mass Transfer, 2, (2001), 167.

9. Muthucumaraswamy, R.: Effect of a chemical reaction on a moving isothermal vertical surface with suction. Acta Ciencia Indica, 155, (2002) 65-70.

10. Sharma, P. R. and Singh, G.: Effects of variable thermal conductivity and heat source/sink on MHD flow near a stagnation point on a linearly stretching sheet. Journal of Applied Fluid Mechanics, 2, (2008), 13-21.

11. Devi, R. L. V. R., Poornima, T., Reddy, N. B. and Venkataramana, S.: Radiation and mass transfer effects on MHD boundary layer flow due to an exponentially stretching 
sheet with heat source. International Journal of Engineering and Innovative Technology, 3(8), (2014), 33-38.

12. Gangadhar, K. and Bhaskar, R. N.: Chemically reacting MHD boundary layer flow of heat and mass transfer over a moving vertical plate in a porous medium with suction. Journal of Applied Fluid Mechanics, 6(1), (2013), 107-114.

13. Yıн, K. A.: Uniform suction/blowing effect on force convection about wedge. Acta Mechanica, 128, (1998), 173-181.

14. Watanabe, T.: Thermal boundary layer over a wedge with suction or injection in force flow. Acta Mechanica, 83, (1990), 119-126.

15. Bhattacharyya, K.: Effects of heat source/sink on MHD flow and heat transfer over a shrinking sheet with mass suction. Chemical Engineering Research Bulletin, 15, (2011), $12-17$.

16. AJADi, S. O.: A note on the unsteady flow of dusty viscous fluid between two parallel plates. Journal of Applied Mathematics and Computing, 18(1), (2005), 393-403.

17. Dulal, P. and Hiranmoy, M.: Influence of temperature-dependent viscosity and thermal radiation on MHD forced convection over a no-isothermal wedge. Applied Mathematics and Combustion, 212, (2009), 194-208.

18. Baoheng, Y.: Approximate Analytical Solution to the Falkner-Skan wedge flow with the permeable wall of uniform suction. Commun Nonlinear Sci Numer Simulat, 14, (2009), 3320-3326.

19. Ajadi, S. O. and Zuilino, M.: Approximate analytical solutions of reaction-diffusion equations with exponential source term; Homotopy perturbation method. Applied Mathematics letters, 24, (2011), 1634-1639.

20. AJADI, S. O.: Approximate analytical solution for critical parameters in a thermal explosion problem. Applied Mathematics and combustion, 218, (2011), 2005-2010.

21. Taghipour, R.: Application of Homotopy perturbation method on some linear and nonlinear parabolic equations. International Journal of Recent Research and Applied Studies, 6(1), (2011), 55-59. 